\title{
TRAFAIR: Análisis de los flujos de tráfico para mejorar la calidad del aire urbano
}

\author{
Lorena Marrodán ${ }^{1}$, Raquel Trillo², Javier Fabra², Javier Nogueras ${ }^{3}$, María U. Alzueta ${ }^{1}$ \\ ${ }^{1}$ Grupo de Procesos Termoquímicos (GPT) \\ ${ }^{2}$ Grupo de I+D en Computación Distribuida (DisCo) \\ ${ }^{3}$ Grupo de Sistemas de Información Avanzados (IAAA) \\ Instituto de Investigación en Ingeniería de Aragón (I3A) \\ Universidad de Zaragoza, Mariano Esquillor s/n, 50018, Zaragoza, Spain. \\ Tel.+34-976762707, e-mail: marrodan@unizar.es
}

\section{Resumen}

El proyecto TRAFAIR reúne a 9 socios de España e Italia para desarrollar servicios innovadores empleando datos de calidad del aire, condiciones meteorológicas y de flujos de tráfico, en beneficio tanto de los ciudadanos, como de las autoridades responsables de la toma de decisiones que afectan a la calidad del aire.

\section{Introducción}

En la actualidad, la contaminación del aire, como consecuencia de las elevadas emisiones de contaminantes atmosféricos, es la primera causa de muerte, debida a causas ambientales, en Europa y un importante problema de salud a nivel mundial. Según la Agencia Internacional de Energía, la mala calidad del aire es la causa de aproximadamente 6.5 millones de muertes cada año. Como un paso hacia la mejora de la calidad del aire, la Comisión Europea (CE), en 2013, puso en marcha el Programa Clean Air [1]. En este programa se definen una serie de políticas específicas para que los estados miembros garantizacen una buena calidad del aire para sus ciudadanos. Actualmente la situación es crítica en algunos estados miembros, tal es así, que, en 2017, la CE advirtió a 5 países, entre ellos España e Italia, por continuos episodios de contaminación atmosférica, siendo el tráfico urbano una de las principales fuentes de emisión de dichos contaminantes.

En este contexto, actualmente, gran parte de las administraciones públicas y los ciudadanos carecen de herramientas para estimar el nivel de contaminación a escala urbana como resultado de diferentes condiciones en los flujos de tráfico, optimizar las estrategias de control y aumentar la conciencia social sobre la calidad del aire.

El proyecto TRAFAIR persigue desarrollar este tipo de herramientas creando un marco común para la monitorización del tráfico y de la calidad del aire urbano en tiempo real, así como para el pronóstico de la dispersión de la contaminación para las próximas 24 ó 48 horas. En concreto, se persigue implantar estas herramientas en 6 ciudades europeas (Santiago de Composterla, Zaragoza, Módena, Pisa, Florencia y Liborno). A continuación, se describe la metodología prevista para el caso concreto de la ciudad de Zaragoza.

\section{Metodología}

El proyecto TRAFAIR incluye dos metodologías diferentes. Por un lado, el uso de sensores de bajo coste distribuidos por el área urbana y calibrados a partir de los datos de la red de estaciones del Ayuntamiento de Zaragoza para la medición de contaminantes atmosféricos. Por otro lado, el uso de métodos de simulación para construir un modelo de tráfico urbano basado en datos en tiempo real, para posteriormente proporcionar el pronóstico de la calidad del aire urbano basado en los pronósticos meteorológicos y los flujos de tráfico previstos. La Figura 1 muestra una visión general del proyecto TRAFAIR en su conjunto.

La entrada principal de datos estará constituida por los flujos de tráfico (en el caso de la ciudad de Zaragoza, proporcionados por la red de estaciones permanentes del Ayuntamiento), datos meteorológicos y del pronóstico del tiempo (proporcionados por AEMET), la composición de la flota de vehículos de la ciudad de Zaragoza (estos datos han sido extraídos del sitio Web de la Dirección General de Tráfico, DGT) y el modelo en 3D del área urbana (datos obtenidos a partir del catastro municipal). Para el cálculo de las emisiones de las diferentes categorías de vehículos, se utiliza la metodología y los factores de emisión propuestos por la Agencia Europea de Medio Ambiente (EEA) [2].

Los datos de entrada serán utilizados por el modelo de tráfico, para producir mapas de tráfico vehicular y, por el modelo de dispersión de la contaminación 
del aire, para proporcionar un pronóstico diario de la calidad del aire dentro del área urbana. Para el cómputo de la dispersión atmosférica se utilizará el modelo lagrangiano GRAL, de código de acceso libre y que ha sido desarrollado, en los últimos años, por la Universidad de Graz (Austria) [3].

Los resultados de predicción obtenidos por los diferentes modelos, además de los datos de observación de los diferentes sensores que se instalarán en el marco del proyecto, se publicarán en portales de datos abiertos de las 6 ciudades. Para ello, se seleccionarán estándares relevantes relacionados con las directivas INSPIRE para la creación de Infraestructura de Datos Espaciales (IDE) de Europa, y con la Web de Datos. En concreto, para el acceso a metadatos se considerarán los estándares Catalog Service del OGC (Open Geospatial Consortium) y Data Catalog Vocabulary del W3C (World Wide Web Consortium).

En la etapa final del proyecto, se desarrollarán aplicaciones de usuario final tanto para los ciudadanos como para las diferentes administraciones públicas de las ciudades involucradas en el proyecto. Dichas aplicaciones tienen como objetivo principal aumentar la conciencia pública sobre la contaminación del aire urbano, y ofrecer a las diferentes administraciones públicas sistemas de monitorización y simulación de la calidad del aire y tráfico para facilitar la definición de estrategias y medidas a implatar relativas a la gestión del tráfico.

\section{Conclusiones}

El proyecto TRAFAIR tiene como objetivo estimar el nivel de contaminación a escala urbana, mediante el desarrollo de un servicio para realizar pronósticos de la calidad del aire en función de las previsiones meteorológicas y de los flujos de tráfico.

Los resultados obtenidos enriquecerán el Portal de Datos Europeo (European Data Portal) al agregar nuevos conjuntos de datos de calidad del aire en forma de mapas, en lugar de proporcionar datos puntuales (como en el caso de las estaciones legales de calidad del aire ya disponibles). Dichos mapas describirán los niveles de contaminación dentro de una ciudad, estimulando así el interés de los ciudadanos que trabajan, viven o transitan en esa zona específica.

\section{REFERENCIAS}

[1]. https://eur-lex.europa.eu/legalcontent/EN/TXT/PDF/?uri=CELEX:52013DC0918\& from=EN (último acceso mayo de 2019)

[2]. EMEP/EEA, 2016. EEA air pollutant emission inventory guidebook. Actualizado en Julio de 2018. Disponible en internet en https://www.eea.europa.eu/publications/emep-eeaguidebook-2016 (último acceso mayo de 2019).

[3]. http://lampz.tugraz.at/ gral/index.php (último acceso mayo de 2019).

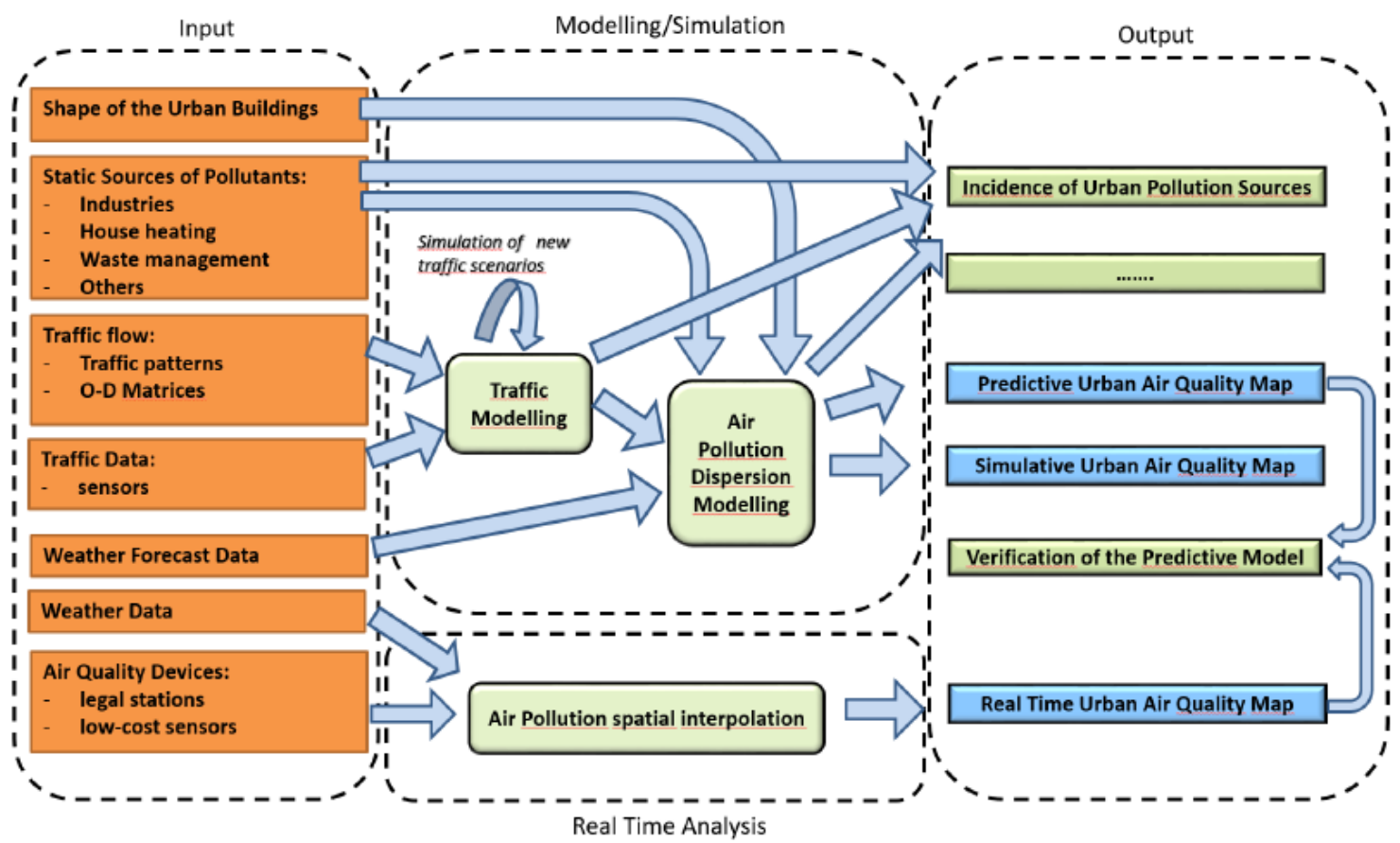

Figura 1. Visión general del proyecto TRAFAIR.

Revista “Jornada de Jóvenes Investigadores del I3A”, vol. 7 (Actas de la VIII Jornada de Jóvenes Investigadores del I3A - 6 de junio de 2019). ISSN 2341-4790. 